\title{
The impact of COVID policies on acute ophthalmology services- experiences from Moorfields Eye Hospital NHS Foundation Trust
}

\author{
Louisa Wickham ${ }^{1} \cdot$ Gordon Hay $^{1} \cdot$ Robin Hamilton $^{1} \cdot$ Jack Wooding $^{1} \cdot$ Harry Tossounis ${ }^{1} \cdot$ Lyndon da Cruz $^{1}$. \\ Dilani Siriwardena ${ }^{1} \cdot$ Nick Strouthidis ${ }^{1}$
}

Received: 23 April 2020 / Revised: 29 April 2020 / Accepted: 1 May 2020 / Published online: 13 May 2020

(c) The Royal College of Ophthalmologists 2020

Early in the national response to the COVID-19 crisis, Moorfields Eye Hospital NHS Foundation Trust (MEH) anticipated that its key contribution to the London health and care system would be to continue to provide safe, effective, eye care to patients requiring sight saving intervention in an environment where many other ophthalmic units would be closed or be unable to access surgical facilities. As a hospital based in London, an epicentre of infection, it was clear that the organisation would rapidly need to respond to the crisis. The overarching premise of this response was that the Trust would move to providing emergency sight- or life-threatening (ocular oncology) care only from March 23rd 2020.

MEH consists of a network of 26 sites across London. It was anticipated that its smaller, peripheral sites would be more susceptible to total closure due to staff sickness or reallocation and/or space requirements of host Trusts. A decision was therefore made to converge critical services to larger or independent sites. Intravitreal injections would be continued at all sites that remained open in recognition of the increased risk and burden of travel on the elderly population that were the majority users of the service. Emergency or urgent surgery would only be continued at the central site and all closed units would be required to redirect appropriate patients to the nearest centre.

MEH developed and used a similar risk stratification approach to that described by ophthalmic professional bodies such as the Royal College of Ophthalmologists and the American Academy of Ophthalmologists as well as in other COVID-19-related articles [1, 2]. Clinical teams were assigned to hot (on site) and cold (off site or

Louisa Wickham

louisa.wickham@moorfields.nhs.uk

1 Moorfields Eye Hospital NHS Foundation Trust, London, UK non-patient facing) working weeks to deliver clinical work and, patient triage and remote consultation, respectively.

Early indications suggested that surrounding general hospitals in Greater London would become overwhelmed with COVID-19 patients and that many ophthalmic units within general hospitals would no longer be able to perform ophthalmic surgery. It was expected that Moorfields would see a sharp rise in patients attending $\mathrm{A}+\mathrm{E}$. Communication networks with other clinical leads were vital in understanding the pressures of neighbouring units. Ophthalmic units were encouraged to continue to triage local emergencies and urgent cases, even if access to theatres for emergency or elective surgery ceased, and only to refer onward to MEH for emergency surgery.

Where possible, patients would be redirected at the front entrance of the unit to use Attend Anywhere, an online consultation service run by the department, for any condition that was not an emergency and, for those deemed to be high risk of being COVI-19 positive from screening questions. Virtual consultation platforms would allow both vulnerable patients and staff members to access and provide clinical care.

Subspecialty stations were created around the hospital to deal with the anticipated increase in ophthalmic emergencies and to allow direct triage of patients into relevant clinics to facilitate early clinical diagnosis and management, avoid re-examination of patients and avoid subsequent unnecessary patient reviews and travel.

Contrary to the anticipated increase in patient numbers either in response to local units closing or reduced outpatient appointments, MEH experienced a significant reduction in total attendance numbers. Prior to the lockdown, the A\&E department would manage an average of 1900 cases per week, this reduced by $>50 \%$ (Table 1 ). Low attendance rates for face-face appointments enabled swift triage and management with an average wait time of $1 \mathrm{~h}$ from registration to discharge in contrast to the 
Table 1 Total attendances at Moorfields $\mathrm{A}+\mathrm{E}$ (face to face and Attend Anywhere), City Road for week commencing 15th March 2020 as compared with 2019.

\begin{tabular}{lllllll}
\hline A + E attendances & Date & & & & & \\
\hline 2020 & $15 / 03$ & $22 / 03$ & $29 / 03$ & $05 / 04$ & $12 / 04$ & $19 / 04$ \\
& 1371 & 833 & 502 & 517 & 673 & 714 \\
2019 & $17 / 03$ & $24 / 03$ & $31 / 03$ & $07 / 04$ & $14 / 04$ & $21 / 04$ \\
& 1960 & 1911 & 1868 & 1861 & 1943 & 1808 \\
& $-30 \%$ & $-56 \%$ & $-73 \%$ & $-72 \%$ & $-65 \%$ & $-61 \%$ \\
\hline
\end{tabular}

Table 2 Top five A + E presentations before and after the initiation of COVID-19 isolation measures and introduction of Attend Anywhere.

\begin{tabular}{|c|c|c|c|}
\hline \multicolumn{2}{|c|}{ Diagnoses for Jan and Feb 2020} & \multicolumn{2}{|c|}{$\begin{array}{l}\text { Diagnoses for March and } \\
\text { April } 2020\end{array}$} \\
\hline Primary diagnosis & Number & Primary diagnosis & Number \\
\hline Blepharitis & 628 & Acute anterior uveitis & 349 \\
\hline $\begin{array}{l}\text { Posterior vitreous } \\
\text { detachment }\end{array}$ & 609 & Corneal abrasion & 343 \\
\hline $\begin{array}{l}\text { Acute anterior } \\
\text { uveitis }\end{array}$ & 606 & $\begin{array}{l}\text { Posterior vitreous } \\
\text { detachment }\end{array}$ & 270 \\
\hline Corneal abrasion & 577 & Blepharitis & 218 \\
\hline Dry eyes & 574 & Chalazion & 191 \\
\hline Total & 2994 & Total & 1371 \\
\hline
\end{tabular}

government target of $4 \mathrm{~h}$. The lower numbers also facilitated appropriate social distancing of patients within the department.

Prior to the COVID-19 outbreak $40 \%$ of attendances to the $\mathrm{A} \& \mathrm{E}$ were not considered to be life- or sight-threatening emergencies. Following implementation of 'at-the-door' triage with digital platform support, the majority of attendances were potentially site threatening conditions. Trauma and painful conditions such as corneal ulcers and orbital cellulitis continued to present to the department (Table 2). Of concern, however, was that some conditions were presenting with more advanced or severe disease, suggesting that patients were reluctant to risk exposure to COVID-19 in order to seek medical attention. The number of trauma patients, particularly those related to domestic abuse, increased.

Digital consultations proved very successful with up to $57 \%$ of cases being managed using Attend Anywhere (Fig. 1). The majority were for minor symptoms managed with reassurance, advice or self-care. Other consultations were for advice following cancellation of outpatient appointments and for prescription requests. $21.1 \%$ required onward referral to the A\&E or to a subspecialty service. This was particularly noteworthy given that the digital platform was only launched within $48 \mathrm{~h}$ of the lockdown, demonstrating how teams can work together proactively and positively to support new initiatives.
In the medical retina service, particular attention was focussed on the importance of maintaining intravitreal injections for the treatment of choroidal neovascular membranes primarily secondary to age-related macular degeneration (ARMD). Existing patients were triaged such that only those most at risk of irreversible visual loss would continue to receive treatment (Fig. 2). Despite limiting the service to high-risk patients only there were multiple patient-initiated cancellations, the average DNA rate increased to $24.9 \%$ (range $13-42.9 \%$ ). Similarly, the number of new presentations of ARMD also decreased significantly (Fig. 3).

An increase in patients presenting for rhegmatogenous retinal detachment (RRD) and emergency surgery was expected due to the closure of surrounding ophthalmic theatres. MEH theatres were configured to provide three emergency theatres and the VR service divided into three teams comprising of three consultants and $2 / 3$ fellows working hot and cold weeks.

Contrary to our expectations, the number of patients presenting with retinal detachment fell significantly following introduction of isolation measures (Fig. 4). The number of retinal detachment operations fell to an average of 14 cases per week, an average drop of $62 \%$ compared with the same period in 2019.

These observations have also been made by VR surgeons in departments across the UK. In an analysis by David Yorston, using data from the Scottish Retinal Detachment Census, 53\% of RD are neither presenting nor being treated (personal communication).

These figures across urgent and emergency ophthalmic services show a worrying trend that patients are neglecting symptoms of visual loss. Although it is possible that the rate of ocular trauma and retinal detachment is actually reduced due to inactivity during isolation, one can also infer that reduced presentation represents concerns regarding the risk of contracting COVID-19, particularly in a perceived highrisk environment such as a hospital. It may also reflect poor awareness of how to access ophthalmic care, many patients are familiar with initially seeking advice from an optometrist or GP. The closure of most optometry practices has limited access to ophthalmic advice. Unfortunately, websites such as NHS 111 advocate visiting an optician if patients have concerns regarding their vision.

In response to this, the ophthalmic community have raised concerns through the Royal College of Ophthalmologists, the Macular Society, NHS 111 and NHS England to increase awareness of the importance of presenting in a timely manner with sight threatening conditions. There has also been increasing media coverage of concerns raised across the medical profession that patients are not presenting or presenting too late with life threatening conditions. Despite this, presentation rates remain well below those 


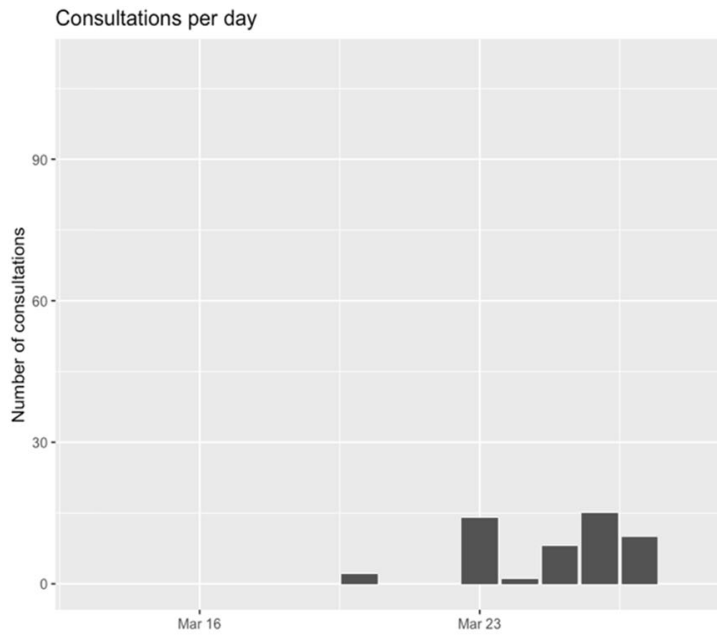

Fig. 1 A bar chart demonstrating the number of Attend Anywhere consultations per day following its active implementation in week commencing March 23rd, 2020. The number of consultations increased dramatically following introduction of isolations measures

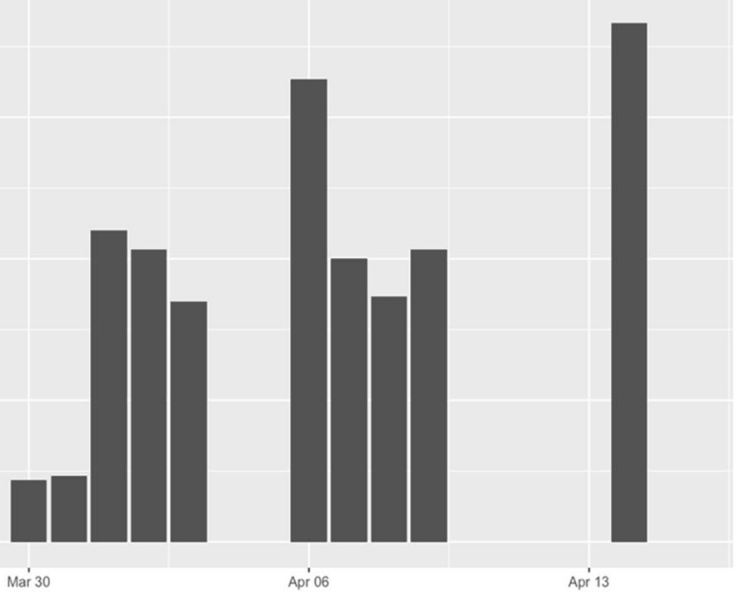

and the use of active triage at the entrance to the department encouraging its use. The Number of Attend Anywhere consultations following its active implementation in week commencing March 23rd, 2020.
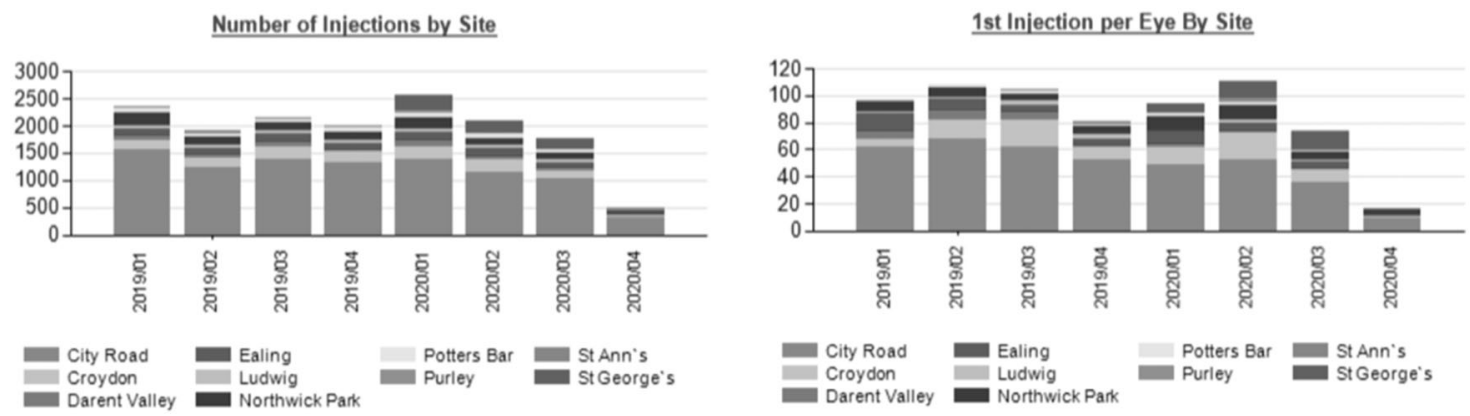

Fig. 2 Bar chart showing the number of injections performed across Moorfields Eye Hospital Trust showing the impact on isolation measures on attendance and first injection rates. Following introduction of isolation measures the number of injections performed in April fell significantly for both follow up and new patients.

Fig. 3 A bar chart showing the number of new AMD cases presenting by week (grey) starting the first week in March 2020 compared with presentation rates in 2019 (orange) and 2018 (blue) for the same week. Week 4 represents the week commencing March 23rd when the Trust mobilised to restrict services to emergency care only, the speed of onset of these measures meant that many patients were not contactable prior to their planned appointment. Despite this numbers still fell significantly as compared to previous years due to high DNA rates. Subsequent weeks show further decreases in patient numbers and represent those with active disease or patients undergoing treatment in their only seeing eye.
Number of new AMD cases by week

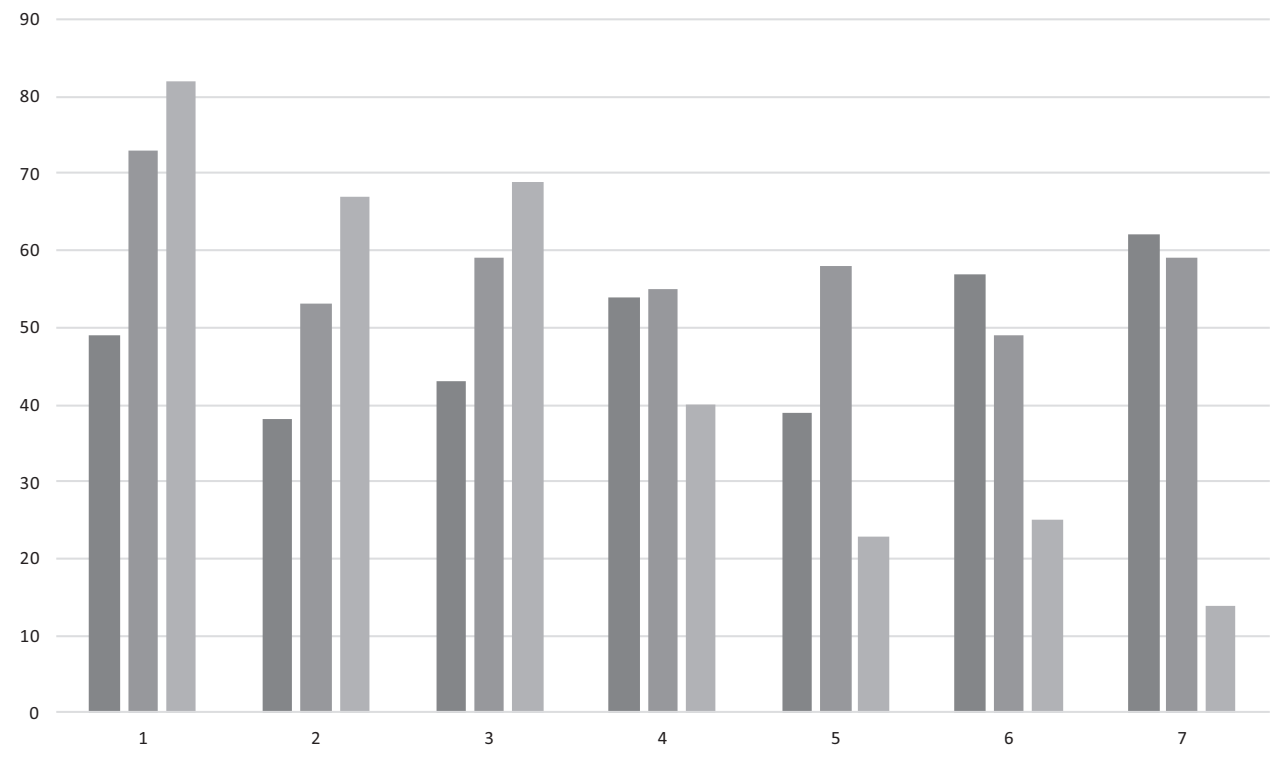




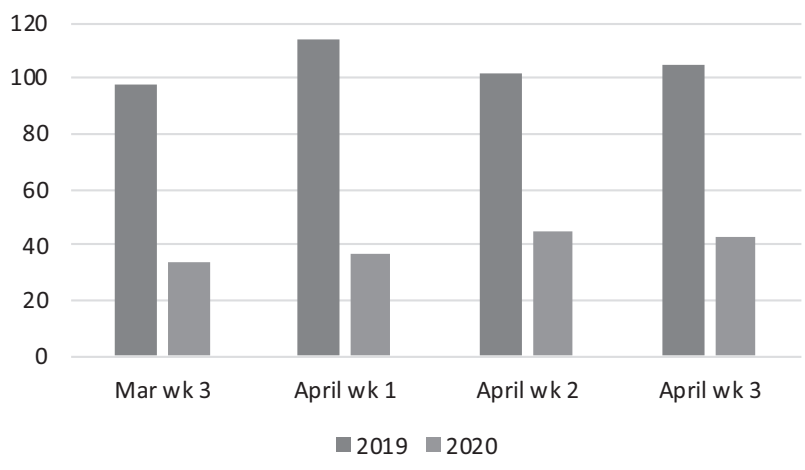

Fig. 4 A bar chart showing the number of patients presenting to VRE in March/April 2019 (blue) and 2020 (orange). The number of patients presenting with retinal detachment fell an average of $62 \%$ compared with same period in 2019.

expected. This has wide ranging implications on the longterm health of our population.

Ophthalmologists need to consider the hidden burden of ophthalmic disease when planning their recovery strategies. It is likely that we will see a considerable increase in emergency presentations which may be more advanced due to delayed presentation as the lockdown is eased. Similarly, when optometry services resume it is likely that we will see a further surge in ophthalmic referrals. It would be prudent to allow for increased emergency work when planning recovery strategies to deal with the potential back-log of known patients.

\section{Compliance with ethical standards}

Conflict of interest The authors declare that they have no conflict of interest.

Publisher's note Springer Nature remains neutral with regard to jurisdictional claims in published maps and institutional affiliations.

\section{References}

1. Shih CK, Chan JCH, Lai JSM. Maintenance of ophthalmic specialist out-patient service during the COVID-19 outbreak: The University of Hong Kong experience. Eye. 2020. https://doi.org/10. 1038/s41433-020-0887-z.

2. Romano MR, Montericcio A, Montalbano C, Raimondi R, Allegrini D, Ricciardelli G, et al. Facing COVID-19 in Ophthalmology department. Curr Eye Res. 2020. https://doi.org/10.1080/02713683. 2020.1752737. 